\title{
A NOTE ON CONTINUOUS COLLECTIONS OF CONTINUOUS CURVES FILLING UP A CONTINUOUS CURVE IN THE PLANE
}

\author{
R. D. ANDERSON AND MARY-ELIZABETH HAMSTROM
}

The purpose of this note is to prove the following

THEOREM. If $G$ is a continuous collection of nondegenerate continuous curves filling up a compact continuous curve in the plane, then with respect to its elements as points $G$ is either an arc or a simple closed curve.

This theorem provides a complement to Theorem VI of [1] which states that if $G$ is a continuous collection of nondegenerate compact continuous curves in the plane which is a compact continuum with respect to its elements as points, then $G$ is a hereditary continuous curve such that the closure of its set of emanation points is totally disconnected.

Some notation similar to that of [1] will be used. A simple chain is a finite collection $x_{1}, x_{2}, \cdots, x_{n}$ of open discs (interiors of simple closed curves) such that $\bar{x}_{i} \cdot \bar{x}_{j}$ exists if and only if $|i-j| \leqq 1$ and is a closed disc (a simple closed curve plus its interior) if it does exist. A (simple) chain $C$ is said to simply cover a set $M$ if $C^{*}$ contains $M$ but for no proper subchain $C^{\prime}$ of $C$ does the closure of $C^{*}$ contain $M .^{1}$ Two chains, $C$ and $C^{\prime}$, are said to be mutually exclusive if $C^{*}$ and $C^{*}$ are mutually exclusive. The chain $C$ is said to be a closed refinement of the chain $C^{\prime}$ provided that each link of $C^{\prime}$ contains the closure of some link of $C$ and the closure of each link of $C$ is contained in some link of $C^{\prime}$.

Proof of TheOREM. We note first that only a countable number of the elements of $G$ contain triods. (See [5].) Denote the elements of some subset of $G$ containing these elements by $g_{1}, g_{2}, \ldots$. Each of the remaining elements of $G$ is an arc or a simple closed curve. If $G$ is not an arc or a simple closed curve with respect to its elements as points, it has an emanation element. Let $H$ denote the closure of the set of emanation elements of $G$. It has been noted that, by Theorem VI of [1], $H$ is totally disconnected.

Let $T$ be an arc of elements of $G$ and suppose that the non-endelement $t$ of $T$ is an isolated element of $T \cdot H$. There is a subarc $T_{1}$ of

Presented to the Society, October 24, 1953; received by the editors October 8 , 1953 and, in revised form, March 10, 1954.

${ }^{1}$ If $G$ is a collection of point sets, $G^{*}$ denotes the sum of its elements. 
elements of $T$ such that $T_{1} \cdot H$ consists of $t$, a non-endelement of $T_{1}$, and possibly one or both of the endelements $t_{1}$ and $t_{2}$ of $T_{1}$. Let $\widetilde{T}_{1}$ be $T_{1}-t_{1}-t_{2}$. Since $G^{*}$ is locally connected, so is $\widetilde{T}_{1}^{*}-t$. If there is a point of $t+t_{1}+t_{2}$ at which $T_{1}^{*}$ is not locally connected then, since $G^{*}$ is locally connected, there exists an arc $s$ in $G^{*}$ which intersects $\widetilde{T}_{1}^{*}-t$ and $t+t_{1}+t_{2}$ with $s \cdot\left(t+t_{1}+t_{2}\right)$ an endpoint of $s$ and with $s$ not a subset of $T_{1}^{*}$. This contradicts the fact that $\tilde{T}_{1}^{*}-t$ and $G^{*}-T_{1}^{*}$ must be mutually separated. Therefore $T_{1}^{*}$ is a continuous curve and it follows easily that $T_{1}^{*}$ has no cut point. Hence from Theorem 54, Chapter IV of [2] it follows that no boundary of a complementary domain of $T_{1}^{*}$ has a cut point and therefore that every such boundary curve is a simple closed curve. Of course no two such boundary curves have more than one point in common. Since $t$ is a non-endelement of $T_{1}$ it contains an interior point of $T_{1}^{*}$ (Theorem 6 and Lemma 1 of [4]) and hence it is not a limit element of $G-T$. Thus $T \cdot H$ contains no isolated element and is a Cantor set if it exists.

Since $G$ is a continuous curve, the set of emanation elements of $G$ lying in $T$ is dense in $T \cdot H$. Since $T \cdot H$ is closed it is not true that both the collection of arcs and the collection of simple closed curves of $T \cdot H$ are dense in $T \cdot H$. (This follows from Theorem I of [1].) Therefore there is a subarc $T_{2}$ of $T$ intersecting $H$ such that the endelements of $T_{2}$ are atriodic non-endelements of $T$ which lie in $T-T \cdot H$ and either $T_{2} \cdot H$ contains no element which is an arc or $T_{2} \cdot H$ contains no element which is a simple closed curve.

Let $V$ be a maximal free $\operatorname{arc}^{2}$ of $T_{2}$ with endelements $a$ and $b$ in $H$. From the argument above (but with $t$ not present) $V^{*}$ must be locally connected. Therefore, as noted above, every complementary domain of $V^{*}$ is bounded by a simple closed curve and no two such boundary curves intersect in more than one point. Since $V$ is a free arc, no more than two of the complementary domains of $V^{*}$ intersect $G^{*}$ $-V^{*}$. Since $G$ is continuous and $a$ and $b$ are limit elements of $T-V$, each of $a$ and $b$ lies in the boundary of a complementary domain of $V^{*}$ and is an arc or a simple closed curve. Since $V^{*}$ is locally connected it follows from the proof of Lemma 1 of [4] or an easy extension of our earlier arguments that each simple closed curve which is an element of $V$ separates $T_{2}^{*}$ in the plane and that if $a$ or $b$-say $a-$ is a simple closed curve and the sequential limiting set of a sequence of elements of $G-V$, at most a finite number of the elements of this sequence lie in the same complementary domain of $a$ as $V^{*}-a$.

2 A free arc in $G$ is an arc of elements of $G$ none of whose non-endelements lies in $H$. By a maximal free arc we shall mean a free arc whose endelements lie in $H$. If $T$ is a subset of $G$ which is an arc, we shall speak of a free arc of $G$ which is a subset of $T$ as a free arc of $T$. 
Suppose $T \cdot H$ contains no arc as an element. Then it follows from the above that all the endelements of maximal free arcs in $T_{2}$ are simple closed curves. It is easy to see from the considerations above that no three such simple closed curves have the property that no one separates the other two in the plane and hence by Lemmas $\mathrm{A}$ and $\mathrm{B}$ of [1] it follows that every element of $T_{2} \cdot H$ is a simple closed curve. But by Lemma C of [1] no element of $T_{2} \cdot H$ can be an emanation element of $G$. Hence we may assume that $T_{2} \cdot H$ has no element which is a simple closed curve.

It follows in a similar manner that only a finite number of the maximal free arcs of $T_{2}$ can contain simple closed curves or, in fact, continuous curves which separate the plane as elements and hence we may consider without essential loss of generality that $T_{2}$ contains no element which separates the plane and which is in a free arc of $T_{2}$.

Since each element $x$ of a free arc of $T_{2}$ is an endelement of some free arc $U$ of $T_{2}$ and thus a subset of a boundary simple closed curve of a complementary domain of $U^{*}, x$ is an arc and it follows from Theorem 3 of [3] that the sum of the elements of every free arc of $T_{2}$ is a closed disc.

Let the element $x_{1}$ of $H$ be an endelement of a maximal free arc $V_{1}$ of $T_{2}$. There are a simple chain $C_{1}$ of link diameter less than 1 and two open discs $D_{1}$ and $D_{2}$ such that (1) $C_{1}^{*}$ does not intersect $g_{1}$ (the first triodic element of $G$ as defined above) and $C_{1}$ simply covers $x_{1}$ but does not cover $V_{1}^{*}$, (2) $C_{1}$ is made up of the links of two simple chains, $C_{11}$ and $C_{12}$, each of which has at least five links and which have only one link in common, (3) if $d$ is an endlink of $C_{1}, \bar{d}-d$ and $x_{1}$ have only one point in common and if $d$ is a non-endlink, $\bar{d}-d$ and $x_{1}$ have only two points in common, (4) $D_{1}$ and $D_{2}$ contain the endpoints of $x_{1},(5)$ for each $i(i=1,2), \bar{D}_{i}-D_{i}$ has only one point in common with $x_{1}$ and $(6)$ the $\bar{D}_{i}(i=1,2)$ lie in distinct endlinks of $C_{1}$ with neither intersecting the closure of any other link of $C_{1}$.

Since $G^{*}$ is locally connected at every point of $x_{1}$ there is a subarc $K_{1}$ of $T_{2}-V_{1}+x_{1}$ containing $x_{1}$ such that every element of $K_{1}$ is covered by $C_{1}$ and intersects both $D_{1}$ and $D_{2}$ and if $A_{1}, A_{2}$, and $A_{3}$ are any consecutive links of $C_{1}$ in that order there is an arc $\tilde{t}$ lying in $G^{*} \cdot\left[A_{2}-A_{2} \cdot \mathrm{Cl}\left(A_{1}+A_{3}\right)\right]$ whose endpoints are separated in $A_{2}-A_{2}$ - $\left(A_{1}+A_{3}\right)$ by the common part of $A_{2}-A_{2} \cdot\left(A_{1}+A_{3}\right)$ and any arc in $K_{1}^{*}$ which intersects both $\bar{A}_{1}$ and $\bar{A}_{3}$. Since there is a domain $U$ of elements of $G$ with $U$ containing $x_{1}$, with the closure of $U^{*}$ lying in $C_{1}^{*}$, and with every element of $U$ containing an arc irreducible between $\bar{D}_{1}$ and $\bar{D}_{2}$, and since every such domain contains elements of $G-T_{2}$, there is a sequence of components of $K_{1}^{*} \cdot\left[C_{1}^{*}-\left(D_{1}+D_{2}\right)\right]$ converging 
to $x_{1}-x_{1} \cdot\left(D_{1}+D_{2}\right)$ each element of which intersects both $\bar{D}_{1}$ and $\bar{D}_{2}$.

There are open discs $D_{1}^{\prime}$ and $D_{2}^{\prime}$ containing $\bar{D}_{1}$ and $\bar{D}_{2}$ respectively such that for each $i(i=1,2), \bar{D}_{i}^{\prime}-D_{i}^{\prime}$ and $x_{1}$ have only one point in common and the $\bar{D}_{i}^{\prime}(i=1,2)$ lie in distinct endlinks of $C_{1}$ with each intersecting the closure of no other link of $C_{1}$. Since $K_{1}$ is continuous and an arc with respect to its elements and each of its elements intersects both $D_{1}$ and $D_{2}$, there is an element $z$ of $K_{1}$ containing two mutually exclusive arcs, $z_{1}$ and $z_{2}$, each irreducible between $\bar{D}_{1}^{\prime}$ and $\bar{D}_{2}^{\prime}$ and lying in different components of $K_{1}^{*} \cdot\left[C_{1}^{*}-\left(D_{1}+D_{2}\right)\right]$. If $z$ belongs to $H$ there is an element $x$ of $H \cdot K_{1}$ which is an endelement of a maximal free arc of $K_{1}$ and contains two mutually exclusive arcs, one intersecting both endlinks of $C_{1}$, the other covered by $C_{11}$ or $C_{12}$ and intersecting both of its endlinks. ${ }^{3}$ If $z$ is not in $H$ it is a nonendelement of a maximal free $\operatorname{arc} Q$ of $K_{1}$. If $Q_{i}(i=1,2)$ denotes the component of $Q^{*} \cdot\left[C_{1}^{*}-\left(D_{1}^{\prime}+D_{2}^{\prime}\right)\right]$ containing $z_{i}, Q_{i}$ contains two arcs, $q_{i 1}$ and $q_{i 2}$, each lying on the boundary simple closed curve of $Q^{*}$ and irreducible between $\bar{D}_{1}^{\prime}$ and $\bar{D}_{2}^{\prime}$. Since if $A_{1}, A_{2}$, and $A_{3}$ are any consecutive links of $C_{1}$ in that order, there is an arc $\tilde{t}$ lying in $G^{*}$ - $\left[A_{2}-A_{2} \cdot \mathrm{Cl}\left(A_{1}+A_{3}\right)\right]$ whose endpoints are separated in $A_{2}-A_{2}$ - $\left(A_{1}+A_{3}\right)$ by every arc of $Q^{*}$ intersecting both $\bar{A}_{1}$ and $\bar{A}_{3}$, none of the $q_{i j}(i, j=1,2)$ contains an arc $t^{\prime}$ which intersects both $\bar{A}_{1}$ and $\bar{A}_{3}$ but does not intersect either endelement of $Q$. This follows from the fact that such a $t^{\prime}$ would lie on the boundary simple closed curve of $Q^{*}$ and contain no limit point of $G^{*}-Q^{*}$, which is inconsistent with the existence of the $\operatorname{arc} \tilde{t}$. Therefore, since each endelement of $Q$ intersects both $\bar{D}_{1}^{\prime}$ and $\bar{D}_{2}^{\prime}$, one such endelement contains two mutually exclusive arcs, one covered by $C_{1}$ and intersecting each of its links, the other covered by either $C_{11}$ or $C_{12}$ and intersecting each of its links.

In any case there is an element $x_{2}\left(\not x_{1}\right)$ of $H \cdot K_{1}$ which is an endelement of a maximal free arc $V_{2}$ of $K_{1}$ and contains two mutually exclusive arcs, $z$ and $z^{\prime}, z$ covered by $C_{1}$ and intersecting each of its links and $z^{\prime}$ covered by a chain $C_{1}^{\prime}\left(=C_{11}\right.$ or $C_{12}$ ) and intersecting each of its links. There are mutually exclusive simple chains $C_{2}$ and $C_{2}^{\prime}$ of link diameter less than $1 / 2$ simply covering $z$ and $z^{\prime}$ respectively, $C_{2}$ a closed refinement of $C_{1}, C_{2}^{\prime}$ a closed refinement of $C_{1}^{\prime}$, and there is a subarc $K_{2}$ of $K_{1}-V_{2}+x_{2}$ containing $x_{2}$ such that every element of it contains two arcs, one covered by $C_{2}$ and intersecting each of its links, the other covered by $C_{2}^{\prime}$ and intersecting each of its links. Thus

${ }^{3}$ Clearly we could, if we chose, require that each of these arcs intersects both endlinks of $C_{1}$. 
by repetition of the above argument, noting that a proper subarc of $x_{1}$ could have been used in lieu of $x_{1}$ and neglecting the chain $C_{2}^{\prime}$ in establishing the chains $C_{3}$ and $C_{3}^{\prime}$, it follows readily that there exist a sequence $C_{2}^{\prime}, C_{3}^{\prime}, \cdots$ of mutually exclusive chains and a sequence $K_{2}, K_{3}, \cdots$ of arcs of elements of $T$ such that (1) $K_{2} \cdot K_{3} \cdots$ is an element $g$ of $G$, (2) for each $i>1, C_{i}^{\prime}$ is a closed refinement of $C_{11}$ or $C_{12}$ of link diameter less than $1 / i$, and (3) $K_{i+1}$ is a subarc of $K_{i}$ not containing $g_{i+1}$ such that if $j \leqq i$, each element of $K_{i+1}$ contains an arc covered by $C_{j}^{\prime}$ and intersecting each of its links. The set $g$ thus contains infinitely many mutually exclusive continua all with diameter greater than some number. But $g$ does not contain a triod and thus is an arc or a simple closed curve. Hence the assumption that $G$ has an emanation point leads to a contradiction and the theorem is proved.

\section{REFERENCES}

1. R. D. Anderson, Continuous collections of continuous curves in the plane, Proc. Amer. Math. Soc. vol. 3 (1952) pp. 647-657.

2. R. L. Moore, Foundations of point set theory, Amer. Math. Soc. Colloquium Publications, vol. 13, 1932.

3. Mary-Elizabeth Hamstrom, Concerning continuous collections of continuous curves, Proc. Amer. Math. Soc. vol. 4 (1953) pp. 240-243.

4. - Concerning webs in the plane, Trans. Amer. Math. Soc. vol. 74 (1953) pp. 500-513.

5. R. L. Moore, Concerning triods in the plane and the junction points of plane continua, Proc. Nat. Acad. Sci. U.S.A. vol. 14 (1928) pp. 85-88.

University of Pennsylvania and

Goucher College 